\title{
Analysis of potentially inappropriate medications (PIM) used in elderly outpatients in departments of internal medicine by using the Screening Tool of Older Persons' Potentially Inappropriate Prescriptions (STOPP) criteria
}

\author{
Qinglian Chen, Lingdi Zhang \\ Pharmacy, Affiliated Xiaoshan Hospital, Hangzhou Normal University, Hangzhou, China \\ Contributions: (I) Conception and design: L Zhang; (II) Administrative support: L Zhang; (III) Provision of study materials or patients: None; (IV) \\ Collection and assembly of data: Q Chen; (V) Data analysis and interpretation: All authors; (VI) Manuscript writing: All authors; (VII) Final approval \\ of manuscript: All authors. \\ Correspondence to: Lingdi Zhang. No. 728, Yucai North Road, Xiaoshan, Hangzhou 311201, China. Email: kristylao@163.com.
}

\begin{abstract}
Background: Potentially inappropriate medications (PIMs) are associated with increased adverse drug reactions (ADRs), admission rate, and mortality rate in elderly patients. Here, we assessed PIM use in elderly outpatients in the departments of internal medicine in our center by using the Screening Tool of Older Persons' potentially inappropriate Prescriptions (STOPP) criteria.
\end{abstract}

Methods: The clinical data of 13,221 outpatients aged $\geq 65$ years in the departments of respiratory medicine, endocrinology, neurology, and cardiovascular medicine of a tertiary hospital from January 2016 to March 2016 were retrospectively analyzed. The incidence of PIM in these patients was assessed by using the STOPP criteria (2014 version). Multivariate logistic regression analysis was performed to identify risk factors for PIM use.

Results: It was found that 1,040 (7.87\%) of 13,221 elderly patients had at least one STOPP-listed PIM, and a total of 1,785 PIMs were identified. The most commonly used PIMs were benzodiazepine, hypnotic Z-drugs (zolpidem), and antipsychotics. Multivariate logistic regression analysis revealed that age [odds ratio $(\mathrm{OR})=1.032,95 \%$ confidence interval $(\mathrm{CI}): 1.022,1.042$ ], gender $(\mathrm{OR}=0.783,95 \% \mathrm{CI}$ : 0.687, 0.892), number of prescribed medications (OR $=1.134,95 \% \mathrm{CI}: 1.106,1.163)$, and number of diagnoses $(\mathrm{OR}=1.450$ 95\% CI: 1.391, 1.510) were significantly associated with PIM use. The incidence of PIM use was highest in the Department of Neurology (12.75\%), followed by the Department of Cardiology (5.90\%), Department of Endocrinology (4.94\%), and Department of Respiratory Medicine (2.90\%). The risk factors for PIMs varied among different departments: except that benzodiazepines and hypnotic Z-drugs (zolpidem) were 2 common PIMs in all departments, the remaining topranked PIMs were department-specific.

Conclusions: PIM use in elderly patients in the outpatient departments of internal medicine is mainly related to age, gender, number of medications, and number of diagnoses. However, it varies among different departments, and the main PIM types are also department-specific. Thus, special attention should be paid to departments with a high incidence and a large number of PIMs, so as to promote rational use of medicines.

Keywords: Potentially inappropriate medication (PIM); Screening Tool of Older Persons' potentially inappropriate Prescriptions (STOPP); elderly outpatients

Submitted Feb 23, 2021. Accepted for publication Apr 21, 2021.

doi: 10.21037/apm-21-799

View this article at: http://dx.doi.org/10.21037/apm-21-799

(c) Annals of Palliative Medicine. All rights reserved. 


\section{Introduction}

The 65-and-older population accounts for $11.94 \%$ of the total population in China, according to the latest data released by the China National Bureau of Statistics in 2018 (1). In addition, up to $78.4 \%$ of the people over 65 years of age in China have chronic diseases, a proportion much higher than that in other age groups (2). Elderly persons are apt to receive multiple drugs for many diseases, which, along with the special physiological status of elderly patients, can easily lead to potentially inappropriate medications (PIMs). First defined by Beers et al. (3) in 1991, PIMs are the drugs for which the effectiveness has not been established and/or the risk of an adverse drug event (ADE) outweighs the expected clinical benefit, particularly when there is no safer alternative therapy for the same condition. Research has shown that the number of diagnoses and the number of prescribed medications are associated with PIM use (4,5). As a result, the incidence of PIM use is particularly high in elderly patients due to their diseases and medications. PIMs may cause adverse drug reactions (ADRs) and lead to ADEs resulting in increased emergency department visits, hospitalizations, mortality, and health care costs $(6,7)$. Therefore, identifying PIMs is clinically important.

The Beers criteria and the Screening Tool of Older Persons' potentially inappropriate Prescriptions (STOPP) criteria are 2 valuable tools for detecting PIM. The Beers criteria have been widely used, and most Chinese studies on PIM in elderly patients also adopt the Beers criteria. Proposed by a research group at Cork University Hospital, Ireland, the STOPP criteria is a screening tool for PIM in elderly populations. Since the publication of the first edition of STOPP criteria in 2008, it has been used in more than 20 countries for evaluating clinical research and practices of irrational medication use in the elderly. It can effectively evaluate PIM use, strengthen the regulation of drug abuse, and reduce the incidence of ADEs in the elderly. In 2014, an expert panel updated the criteria, resulting in an overall $31 \%$ expansion of STOPP criteria (8). One study found that the STOPP criteria set was more sensitive than the Beers criteria set, as it could identify more PIMs and had a stronger correlation with ADEs (9). In China, most studies investigating PIMs in elderly patients are still using the Beers criteria, and the STOPP criteria are less often used. Most PIM studies have been performed in inpatients $(10,11)$, and few studies have assessed PIM use in outpatients by using the STOPP criteria. Compared with the number inpatients, the number of outpatients is larger in and can better reflect the actual long-term medication use in elderly patients. Here, we evaluated the incidence of PIM use in elderly outpatients in the internal medicine departments at our center and explored the risk factors for PIMs by using the STOPP criteria (2014 version). We present the following article in accordance with the STROBE reporting checklist (available at http://dx.doi.org/10.21037/apm-21-799).

\section{Methods}

\section{Study design}

By using the STOPP criteria (2014 version), we analyzed the PIMs and their influencing factors in elderly outpatients in the departments of respiratory medicine, endocrinology, neurology, and cardiology in a tertiary hospital. We further assessed the variability of PIM use in different departments and diagnosed diseases, with an attempt to inform and promote the rational use of drugs in elderly patients.

The prescription data (including patient number, prescription date, department, gender, age, diagnosis, and medications) of elderly patients (age $\geq 65$ years) in the 4 major internal medicine departments (i.e., the departments of respiratory medicine, endocrinology, neurology, and cardiology) at a tertiary hospital from January to March 2016 were exported using the Rational Drug Use software. The inclusion criteria for participants were age $\geq 65$ years and having used at least one drug. The exclusion criteria included incomplete treatment or medication records. The judgment of PIMs was based on the STOPP criteria 2014 edition, which includes 12 sections (including Indication of Medication, Cardiovascular System, Antiplatelet/ Anticoagulant Drugs, and Central Nervous System and Psychotropic Drugs) and 80 criteria items. As the creatinine clearance rate was not retrieved from our system, the "Renal System" section was excluded, and finally 74 prescriptions were included the final analysis. The study was conducted in accordance with the Declaration of Helsinki (as revised in 2013). The study was approved by the Ethics Committee of Xiaoshan Hospital (Hangzhou, China). Individual consent for this retrospective analysis was waived.

\section{Statistical methods}

The statistical analysis was completed in SPSS 19.0 software package (IBM Corp.). The measurement data are presented as mean standard deviations $(\bar{x} \pm \mathrm{SD})$ and were compared 
Table 1 General data of the patients

\begin{tabular}{|c|c|c|c|c|c|}
\hline Item & Total & $\begin{array}{c}\text { Department of } \\
\text { Respiratory Medicine }\end{array}$ & $\begin{array}{l}\text { Department of } \\
\text { Endocrinology }\end{array}$ & $\begin{array}{l}\text { Department of } \\
\text { Neurology }\end{array}$ & $\begin{array}{c}\text { Department of } \\
\text { Cardiology }\end{array}$ \\
\hline No. & 13,221 & 2,156 & 1,317 & 5,185 & 4,563 \\
\hline Men & 6,787 & 1,202 & 633 & 2,572 & 2,380 \\
\hline Women & 6,434 & 954 & 684 & 2,613 & 2,183 \\
\hline Number of diagnoses $(\bar{x} \pm \mathrm{SD})$ & $2.12 \pm 1.30$ & $1.38 \pm 0.70$ & $2.49 \pm 1.54$ & $2.14 \pm 1.26$ & $2.35 \pm 1.34$ \\
\hline
\end{tabular}

Table 2 Incidence of PIMs in total and in different departments

\begin{tabular}{lccc}
\hline Department & PIM group & Non-PIM group & Incidence of PIMs \\
\hline Total & 1,040 & 12,181 & $7.87 \%$ \\
$\begin{array}{l}\text { Department of Respiratory } \\
\text { Medicine }\end{array}$ & 45 & 2,111 & $2.09 \%$ \\
Department of Endocrinology & 65 & 1,252 & $4.94 \%$ \\
Department of Neurology & 661 & 4,524 & $12.75 \%$ \\
Department of Cardiology & 269 & 4,294 & $5.90 \%$ \\
\hline
\end{tabular}

PIM, potentially inappropriate medication.

using the independent samples $t$-test. The count data are presented using cases and percentages and were compared using the chi-square test. The correlation analysis was performed by using the multivariate logistic regression method, and the results are expressed as the odds ratio (OR) and $95 \%$ confidence interval $(95 \% \mathrm{CI})$. A P value of $<0.05$ was considered statistically significant.

\section{Results}

\section{The general data}

According to the inclusion and exclusion criteria, a total of 13,221 patients were included in this study, including 2,156 from the Department of Respiratory Medicine, 1,317 from the Department of Endocrinology, 5,185 from the Department of Neurology, and 4,563 from the Department of Cardiology.

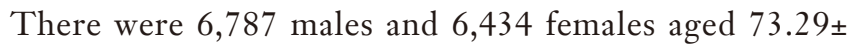
6.53 years (range, 69-102) years. The average number of prescribed medicines was $3.2 \pm 2.52$, and the average number of diagnoses (which referred to the number of diseases diagnosed in a single prescription) was $2.12 \pm 1.3$ (Table 1).

\section{Incidence of PIM use}

STOPP identified 1,040 PIMs affecting 1,040 (7.87\%) of 13,221 patients, among whom 45 were from the Department of Respiratory Medicine, 65 from the Department of Endocrinology, 661 from the Department of Neurology, and 269 from the Department of Cardiology. The incidence of PIM use was highest in the Department of Neurology, followed by the Department of Cardiology, the Department of Endocrinology, and the Department of Respiratory Medicine (Table 2).

\section{Results of logistic regression analysis of risk factors for PIMs}

The patients were then divided into a PIM group and nonPIM group according to the use of PIMs. The mean age, mean number of prescribed medications, mean diagnoses, and proportion of females were $75 \pm 7.04$ years, $3.51 \pm 3.44$, $2.95 \pm 1.66$, and $52.98 \%$, respectively, in the PIM group, which were significantly different from those in the nonPIM group $(73.14 \pm 6.47$ years, $2.51 \pm 1.84,2.05 \pm 1.24$, and 
Table 3 Univariate analysis of the risk factors for PIMs

\begin{tabular}{lccc}
\hline Item & PIM group & Non-PIM group & P value \\
\hline Age $( \pm$ S, years $)$ & $75 \pm 7.04$ & $73.14 \pm 6.47$ & 0.000 \\
Gender & & & 0.004 \\
Men & $489(47.02 \%)$ & $6,298(51.70 \%)$ & $5,883(48.30 \%)$ \\
Women & $551(52.98 \%)$ & $2.51 \pm 1.84$ & 0.000 \\
Number of prescribed medications $(\bar{x} \pm \mathrm{SD})$ & $3.51 \pm 3.44$ & $2.05 \pm 1.24$ & 0.000 \\
Number of diagnoses $(\bar{x} \pm \mathrm{SD})$ & $2.95 \pm 1.66$ & & \\
\hline
\end{tabular}

PIM, potentially inappropriate medication.

Table 4 Multivariate logistic regression analysis of risk factors for PIMs in total

\begin{tabular}{lcc}
\hline Variables & OR $(95 \% \mathrm{Cl})$ & $\mathrm{P}$ value \\
\hline Age $( \pm \mathrm{S}$, years $)$ & $1.032(1.022-1.042)$ & 0.000 \\
Gender & $0.783(0.687-0.892)$ & 0.001 \\
Number of prescribed medications $(\bar{x} \pm \mathrm{SD})$ & $1.134(1.106-1.163)$ & 0.000 \\
Number of diagnosis $(\bar{x} \pm \mathrm{SD})$ & $1.450(1.391-1.510)$ & 0.000 \\
\hline
\end{tabular}

Age: age is set as the independent variable. Gender: $\operatorname{man}=1$, woman $=0$. Number of prescribed medications: the number of medication types set as the independent variable. Number of diagnoses: the number of diagnoses set as the independent variable. PIM use: yes $=1$, no $=0 . \mathrm{Cl}$, confidence interval; OR, odds ratio.

$48.30 \%$, respectively; Table 3).

Multivariate logistic regression analysis was performed with PIM use as the dependent variable, while age, gender, number of diagnoses, and number of prescribed medications were the independent variables used to investigate the risk factors associated with PIM use. It was found that age, gender, number of prescribed medications, and number of diagnoses were all significantly associated with PIM use, among which age, number of diagnoses, and number of prescribed medications were positively correlated with PIM use, with women being more likely to use PIMs than men (Table 4).

As shown in Table 5, age, gender, number of diagnoses, and number of prescribed medications showed different correlations with PIM use in different outpatient departments. Age was positively associated with PIM use in all 4 departments. Although PIM use was not significantly correlated with gender in the Department of Respiratory Medicine and the Department of Endocrinology, female patients were more likely to be PIM users in the Department of Neurology and Department of Cardiology. PIM use was not significantly correlated with the number of prescribed medications in the Department of
Endocrinology; however, a larger number of prescribed medications was associated with higher risk of PIM use in the Department of Respiratory Medicine and the Department of Neurology; in contrast, a smaller number of prescribed medications was associated with higher risk of PIM use in the Department of Cardiology. Finally, the number of diagnoses was positively correlated with PIM use in all the 4 departments.

\section{PIM types and distribution}

A total of 1,785 PIMs were identified in the PIM group ( $\mathrm{n}=1,040$, with a maximum of 13 PIMs in a single patient). The most frequently detected PIMs were benzodiazepines, followed by hypnotic Z-drugs, and antipsychotics. All 3 medications are drugs that may lead to falls in elderly patients. Other PIMs included the following: (I) long-term aspirin administration at doses greater than $160 \mathrm{mg}$ per day; (II) aspirin use in patients with a past history of peptic ulcer disease without concomitant proton-pump inhibitors (PPIs); (III) aspirin use in patients with a history of peptic ulcer without PPIs, as well as antipsychotic administration (i.e., other than quetiapine or clozapine) in those with 
Table 5 Multivariate logistic regression analysis of risk factors for PIMs in different departments

\begin{tabular}{|c|c|c|c|c|}
\hline OR, 95\% Cl & $\begin{array}{l}\text { Department of Respiratory } \\
\text { Medicine }\end{array}$ & $\begin{array}{l}\text { Department of } \\
\text { Endocrinology }\end{array}$ & $\begin{array}{l}\text { Department of } \\
\text { Neurology }\end{array}$ & $\begin{array}{l}\text { Department of } \\
\text { Cardiology }\end{array}$ \\
\hline Age $( \pm \mathrm{S}$, years $)$ & $1.062^{*}(1.017-1.109)$ & $1.040 *(1.000-1.081)$ & $1.026^{*}(1.014-1.039)$ & $1.030 *(1.010-1.050)$ \\
\hline Gender & $0.992(0.527-1.865)$ & $1.391(0.826-2.342)$ & $0.808^{*}(0.683-0.955)$ & $0.708^{\star}(0.545-0.919)$ \\
\hline $\begin{array}{l}\text { Number of prescribed } \\
\text { medications }(\bar{x} \pm \mathrm{SD})\end{array}$ & $1.231^{*}(1.092-1.389)$ & $1.018(0.834-1.242)$ & $1.124^{*}(1.093-1.156)$ & $0.730^{\star}(0.652-0.817)$ \\
\hline
\end{tabular}

Age: age is set as the independent variable. Gender: $\operatorname{man}=1$, woman $=0$. Number of prescribed medications: the number of medication types set as the independent variable. Number of diagnoses: the number of diagnoses set as the independent variable. PIM use: yes $=1$, no $=0$. * $\mathrm{P}<0.05$. Cl, confidence interval; OR, odds ratio.

parkinsonism or Lewy body dementia; (IV) nonsteroidal anti-inflammatory drugs (NSAIDs) use in patients with severe hypertension; and (V) NSAIDs other than COX2 selective agents in patients with a history of peptic ulcer disease or gastrointestinal bleeding, unless administered with concurrent PPI or $\mathrm{H}_{2}$ antagonist (see Table 6).

Both benzodiazepines and benzodiazepines, which can increase the risk of falls in elderly patients, were among the top PIMs in all 4 departments. The other top ranking PIMs were department-specific. The main PIMs in the Department of Respiratory Medicine also included theophylline as a monotherapy for chronic obstructive pulmonary disease (COPD); the main PIMs in the Department of Endocrinology also included longacting sulfonylureas for patients with type 2 diabetes mellitus (T2DM) and beta-blockers in diabetes mellitus with frequent hypoglycemic episodes; the main PIMs in the Department of Neurology also included antipsychotics and long-term use of aspirin enteric-coated tablets at doses greater than $160 \mathrm{mg} / \mathrm{d}$; the main PIMs in the Department of Cardiology also included NSAIDs (including NSAIDs for patients with severe hypertension), nonselective cyclooxygenase-2 (COX-2) inhibitors for patients with peptic ulcers or bleeding without the combined use of PPIs, and aspirin use in patients with a history of peptic ulcer without PPUs,

\section{Discussion}

\section{Incidence of PIM use}

According to the STOPP criteria (2014 edition), the incidence of PIM use in elderly patients in the 4 outpatient departments was $7.87 \%$ in our current study. It was found that the incidence of PIM use was highest in the Department of Neurology and lowest in the Department of Respiratory Medicine. Only a limited number of studies have investigated the incidence of PIM use in outpatients using the STOPP criteria. Paksoy et al. evaluated PIM use at an outpatient oncology clinic in Istanbul, Turkey, using the STOPP criteria, and found that the incidence of PIM use was $15.79 \%$ (12). Weng et al. from Taiwan Province of China used STOPP criteria to evaluate PIM in elderly patients with chronic diseases in a hospital and found that the incidence of PIM use was 39\% (13). Compared with the above 2 studies, the incidence of PIM use was relatively low in our current study, which may be explained by the smaller number of prescribed medications among our patients. The proportion of patients using $\geq 5$ prescribed medications reached $94.73 \%$ in the Turkish study, and the average number of medications used by patients in the study performed by Weng et al. was $6.7 \%$. In contrast, the number of prescribed medications was much lower in our current study (mean 3.2). The difference in the incidences of PIM use is mainly related to the different number of prescribed medications and the different number of diagnoses, which of course needs to be validated in more studies on PIM use in outpatients by using the STOPP criteria.

\section{Risk factors of PIM use}

Multivariate logistic regression analysis in our current study showed that age, gender, number of diagnoses, and number of prescribed medications were all significantly associated with PIM use. Among these factors, age, number of diagnoses, and number of prescribed medications were positively correlated with PIM use, with women being more likely to be PIM users than men. Many STOPP criteria- 
Table 6 Distribution and total cases of PIM in total (8)

STOPP criteria

PIM cases, $n(\%)$

Cardiovascular system

Beta-blocker in combination with verapamil or diltiazem (risk of heart block)

Beta-blocker for bradycardia (<50 beats/min), type II atrioventricular block or complete atrioventricular block (risk

of complete atrioventricular block and cardiac arrest)

Thiazide diuretic in patients with history of gout (risk of exacerbating hypokalemia, hyponatremia, hypercalcemia, and gout)

Anticoagulants and antiplatelets

Long-term aspirin use at doses greater than 160mg per day (increased risk of bleeding, no evidence for increased efficacy)

Aspirin use with a past history of peptic ulcer disease without concomitant PPIs (risk of recurrent peptic ulcer)

Aspirin, clopidogrel, dipyridamole, vitamin $\mathrm{K}$ antagonists, direct thrombin inhibitors, or factor Xa inhibitors with concurrent significant bleeding risk; i.e., uncontrolled severe hypertension, bleeding diathesis, recent nontrivial spontaneous bleeding (high risk of bleeding)

Antiplatelet agents with vitamin $\mathrm{K}$ antagonist, direct thrombin inhibitor, or factor Xa inhibitors in patients with stable coronary, cerebrovascular or peripheral arterial disease (no added benefit from dual therapy)

Vitamin $\mathrm{K}$ antagonist or direct thrombin inhibitor for first pulmonary embolus without continuing provoking risk factors (e.g., thrombophilia)

NSAIDs and vitamin $\mathrm{K}$ antagonists, direct thrombin inhibitors or factor Xa inhibitors in combination (risk of major gastrointestinal bleeding)

NSAIDs with concurrent antiplatelet agent(s) without PPI prophylaxis (increased risk of peptic ulcer disease)

Central nervous system and psychotropic drugs

Tricyclic antidepressants (TCAs) with dementia, narrow angle glaucoma, cardiac conduction abnormalities, prostatism, or prior history of urinary retention (risk of worsening these conditions)

Benzodiazepines used for $\geq 4$ weeks (no indication for longer treatment; risk of prolonged sedation, confusion, impaired balance, falls, road traffic accidents; all benzodiazepines should be withdrawn gradually if taken for more than 4 weeks as there is a risk of causing a benzodiazepine withdrawal syndrome if stopped abruptly)

Antipsychotics (i.e., other than quetiapine or clozapine) used in those with parkinsonism or Lewy body dementia (risk of severe extra-pyramidal symptoms)

Anticholinergics/antimuscarinics to treat extra-pyramidal side effects of neuroleptic medications (risk of anticholinergic toxicity)

Neuroleptics used as hypnotics, unless sleep disorder is due to psychosis or dementia (risk of confusion, hypotension, extra-pyramidal side effects, falls)

First-generation antihistamines (safer, less toxic antihistamines now widely available)

Respiratory system

Theophylline used as monotherapy for COPD (safer, more effective alternatives are available; risk of adverse effects due to narrow therapeutic index)

Musculoskeletal system

NSAIDs other than COX-2 selective agents in patients with a history of peptic ulcer disease or gastrointestinal bleeding, unless applied with concurrent PPIs or $\mathrm{H}_{2}$ antagonists (risk of peptic ulcer relapse)

$65(3.64)$ exacerbation of heart failure)

Table 6 (continued) 
Table 6 (continued)

\begin{tabular}{|c|c|}
\hline STOPP criteria & PIM cases, $n(\%)$ \\
\hline $\begin{array}{l}\text { Antimuscarinic drugs with dementia, chronic cognitive impairment (risk of increased confusion, agitation), } \\
\text { narrow-angle glaucoma (risk of acute exacerbation of glaucoma), or chronic prostatism (risk of urinary retention) }\end{array}$ & $11(0.62)$ \\
\hline $\begin{array}{l}\text { Sulphonylureas with a long duration of action (e.g., glibenclamide, chlorpropamide, glimepiride) in patients with } \\
\text { T2DM (risk of prolonged hypoglycemia) }\end{array}$ & $29(1.62)$ \\
\hline $\begin{array}{l}\text { Beta-blockers in diabetes mellitus with frequent hypoglycemic episodes (risk of suppressing hypoglycemic } \\
\text { symptoms) }\end{array}$ & $8(0.45)$ \\
\hline Hypnotic Z-drugs (e.g., zopiclone, zolpidem, zaleplon; may cause protracted daytime sedation, ataxia) & $259(14.51)$ \\
\hline \multicolumn{2}{|l|}{ Analgesic drugs } \\
\hline $\begin{array}{l}\text { Use of oral or transdermal strong opioids (morphine, oxycodone, fentanyl, buprenorphine, diamorphine, } \\
\text { methadone, tramadol, pethidine, pentazocine) as first-line therapy for mild pain (WHO analgesic ladder not } \\
\text { observed) }\end{array}$ & $40(2.24)$ \\
\hline
\end{tabular}

PIM, potentially inappropriate medication; COPD, chronic obstructive pulmonary disease; NSAID, nonsteroidal anti-inflammatory drug; PPI, proton-pump inhibitor; T2DM, type 2 diabetes mellitus; WHO, World Health Organization.

based studies have shown that PIM use is associated with the number of diagnoses and the number of prescribed medications (12-15), which was consistent with our findings; however, the roles of gender and age remained controversial. Studies in Taiwan Province of China, Kuwait, and Japan suggested gender was not associated with PIM use $(13,16,17)$, and studies in Turkey, Taiwan Province of China, Japan, Spain, and Malaysia concluded that age was not associated with PIM use $(12,13,16-18)$; in contrast, another study in mainland China revealed that PIM use was related with both age and gender (5). Quite a few studies in mainland China showed that PIM use was associated with age or with both age and gender, which was quite different from reports in other countries and regions. Our current study also showed that the risk factors for PIM use varied across different departments. Although the these risk factors differed from those reported in other studies $(13,16)$, the number of prescribed medications and the number of diagnoses were positively associated with PIM use, except for in the Department of Cardiology.

\section{Major PIM types and their distribution}

The most frequent PIM in our current study was benzodiazepines (46.89\%), followed by hypnotic Z-drugs (14.51\%), and antipsychotics $(9.58 \%)$. Other relatively common PIM types included long-term aspirin at doses greater than $160 \mathrm{mg}$ per day $(4.03 \%)$ and aspirin with a past history of peptic ulcer disease without concomitant PPIs $(2.75 \%)$, which was basically consistent with other studies $(9,16,17)$. Apart from benzodiazepines and hypnotic Z-drugs (zolpidem) being the 2 common PIMs in all departments, the remaining top PIMs were departmentspecific. In the Department of Respiratory Medicine, theophylline as monotherapy for COPD was among the top PIMs. In the Department of Endocrinology, long-acting sulfonylureas for type 2 diabetes mellitus (T2DM) patients was a common PIM; notably, the use of glibenclamide was the most frequent PIM in outpatients in a Nigerian study (15), which might be due to the high proportion of T2DM patients among the outpatients. In the Department of Neurology, antipsychotics and long-term use of $>160 \mathrm{mg}$ 
aspirin were the major PIMs, which is related to the disease types in the Department of Neurology where aspirin is often used as a first-line drug for secondary prevention of cerebral infarction. Parkinson disease is also a neurological disease, and psychiatric symptoms are one of its nonmotor symptoms. A variety of antipsychotics are used in Parkinson disease patients, which is likely to aggravate Parkinson disease due to the extrapyramidal toxicities of these drugs. Furthermore, antipsychotics often cause falls in elderly patients. In the Department of Cardiology, NSAIDs for severe hypertension and NSAIDs other than COX-2 selective agents in patients with a history of peptic ulcer disease or gastrointestinal bleeding without concurrent PPIs ranked the third and fourth most common PIMs. Abegaz et al. (19) analyzed the PIMs in inpatients with cardiovascular diseases and found that the most common PIM was aspirin in combination with vitamin $\mathrm{K}$ antagonist in patients with chronic atrial fibrillation, which, however, was less common among out outpatients in the Department of Cardiology. Therefore, some PIMs (e.g., benzodiazepines) are commonly used in elderly outpatients in the internal medicine departments; however, the topranked PIMs vary across different departments and differ between outpatient clinics and inpatient wards. The medication habits also differ among different hospitals. Thus, the distribution of PIMs has its unique characteristics in different medical institutions.

\section{Conclusions}

In our current study, the incidence of PIMs among elderly outpatients was $7.87 \%$ according to the STOPP criteria. The main risk factors for PIM use were age, gender, number of prescribed medications, and number of diagnoses; the major PIMs were benzodiazepines, hypnotic Z-drugs, antipsychotics, and other drugs that increase the fall risk. The incidence of PIM use was highest in the Department of Neurology, and thus it is particularly important to prevent PIM use in this department. The risk factors for PIM use vary among different departments, which may be explained by the different underlying conditions of the outpatients. The distribution of PIMs is department-specific, although there are some shared PIMs. The corresponding preventive measures should be taken in these departments.

Our current study also confirmed that STOPP criteria could not only measure the incidence of PIMs but also identify the specific types of PIM in outpatients. Thus, it is a valuable tool for assessing prescriptions and identifying possible PIMs in elderly patients and deserves further application in clinical settings.

\section{Acknowledgments}

Funding: Supported by 2020 Zhejiang Provincial Medical and Health Science and Technology Program (Fund No. 2020KY795).

\section{Footnote}

Reporting Checklist: The authors have completed the STROBE reporting checklist. Available at http://dx.doi. org/10.21037/apm-21-799

Data Sharing Statement: Available at http://dx.doi. org/10.21037/apm-21-799

Conflicts of Interest: Both authors have completed the ICMJE uniform disclosure form (available at http://dx.doi. org/10.21037/apm-21-799). The authors have no conflicts of interest to declare.

Etbical Statement: The authors are accountable for all aspects of the work in ensuring that questions related to the accuracy or integrity of any part of the work are appropriately investigated and resolved. The study was conducted in accordance with the Declaration of Helsinki (as revised in 2013). The study was approved by the Ethics Committee of Xiaoshan Hospital (Hangzhou, China). Individual consent for this retrospective analysis was waived.

Open Access Statement: This is an Open Access article distributed in accordance with the Creative Commons Attribution-NonCommercial-NoDerivs 4.0 International License (CC BY-NC-ND 4.0), which permits the noncommercial replication and distribution of the article with the strict proviso that no changes or edits are made and the original work is properly cited (including links to both the formal publication through the relevant DOI and the license). See: https://creativecommons.org/licenses/by-nc-nd/4.0/.

\section{References}

1. National Bureau of Statistics of China. Statistical Communique of the People's Republic of China on the 2016 National Economic and Social Development, 2016.

2. Center for Health Statistics and Information. Analysis 
Report of National Health Services Survey in China. In: Information Center for Health Statistics and Information, editor, 2013.

3. Beers MH, Ouslander JG, Rollingher I, et al. Explicit criteria for determining inappropriate medication use in nursing home residents. UCLA Division of Geriatric Medicine. Arch Intern Med 1991;151:1825-32.

4. Lim YJ, Kim HY, Choi J, et al. Potentially Inappropriate Medications by Beers Criteria in Older Outpatients: Prevalence and Risk Factors. Korean J Fam Med 2016;37:329-33

5. Li H, Pu S, Liu Q, et al. Potentially inappropriate medications in Chinese older adults: The beers criteria compared with the screening tool of older persons' prescriptions criteria. Geriatr Gerontol Int 2017;17:1951-8.

6. Wallace E, McDowell R, Bennett K, et al. Impact of Potentially Inappropriate Prescribing on Adverse Drug Events, Health Related Quality of Life and Emergency Hospital Attendance in Older People Attending General Practice: A Prospective Cohort Study. J Gerontol A Biol Sci Med Sci 2017;72:271-7.

7. Brown JD, Hutchison LC, Li C, et al. Predictive Validity of the Beers and Screening Tool of Older Persons' Potentially Inappropriate Prescriptions (STOPP) Criteria to Detect Adverse Drug Events, Hospitalizations, and Emergency Department Visits in the United States. J Am Geriatr Soc 2016;64:22-30.

8. O'Mahony D, O'Sullivan D, Byrne S, et al. STOPP/ START criteria for potentially inappropriate prescribing in older people: version 2. Age Ageing 2015;44:213-8.

9. Gallagher P, O'Mahony D. STOPP (Screening Tool of Older Persons' potentially inappropriate Prescriptions): application to acutely ill elderly patients and comparison with Beers' criteria. Age Ageing 2008;37:673-9.

10. Hernandez Martin J, Merino-Sanjuan V, Peris-Marti J, et al. Applicability of the STOPP/START criteria to older polypathological patients in a long-term care hospital. Eur J Hosp Pharm 2018;25:310-6.

11. Cruz-Esteve I, Marsal-Mora JR, Galindo-Ortego G,

Cite this article as: Chen Q, Zhang L. Analysis of potentially inappropriate medications (PIM) used in elderly outpatients in departments of internal medicine by using the Screening Tool of Older Persons' Potentially Inappropriate Prescriptions (STOPP) criteria. Ann Palliat Med 2021;10(4):4678-4686. doi: 10.21037/apm-21-799 et al. Potentially inappropriate prescribing in older Spanish population according to STOPP/START criteria (STARTREC study). Aten Primaria 2017;49:166-76.

12. Paksoy C, Ozkan O, Ustaalioglu BB, et al. Evaluation of potentially inappropriate medication utilization in elderly patients with cancer at outpatient oncology unit. J Oncol Pharm Pract 2019;25:1321-7.

13. Weng MC, Tsai CF, Sheu KL, et al. The impact of number of drugs prescribed on the risk of potentially inappropriate medication among outpatient older adults with chronic diseases. QJM 2013;106:1009-15.

14. Awad A, Hanna O. Potentially inappropriate medication use among geriatric patients in primary care setting: A cross-sectional study using the Beers, STOPP, FORTA and MAI criteria. PLoS One 2019;14:e0218174.

15. Fadare JO, Desalu OO, Obimakinde AM, et al. Prevalence of inappropriate medication prescription in the elderly in Nigeria: A comparison of Beers and STOPP criteria. Int J Risk Saf Med 2015;27:177-89.

16. Kimura T, Ogura F, Yamamoto K, et al. Potentially inappropriate medications in elderly Japanese patients: effects of pharmacists' assessment and intervention based on Screening Tool of Older Persons' Potentially Inappropriate Prescriptions criteria ver.2. J Clin Pharm Ther 2017;42:209-14.

17. Nicieza-Garcia ML, Salgueiro-Vazquez ME, JimenoDemuth FJ, et al. Inappropriate prescribing in polypharmacy elderly outpatients taking multiple medications. Are the STOPP criteria useful? Int J Clin Pharmacol Ther 2016;54:172-6.

18. Fahrni ML, Azmy MT, Usir E, et al. Inappropriate prescribing defined by STOPP and START criteria and its association with adverse drug events among hospitalized older patients: A multicentre, prospective study. PLoS One 2019;14:e0219898.

19. Abegaz TM, Birru EM, Mekonnen GB. Potentially inappropriate prescribing in Ethiopian geriatric patients hospitalized with cardiovascular disorders using START/ STOPP criteria. PLoS One 2018;13:e0195949. 